\title{
An Analysis COVID-19 in Mexico: a Prediction of Severity
}

\author{
Marco Ulises Martínez-Martínez, MD, MSc 1,2, Deshiré Alpízar-Rodríguez, MD, PhD', \\ Rogelio Flores-Ramirez, $P h D^{4}$, Diana Patricia Portales-Pérez, PhD $D^{5}$, Ruth Elena Soria-Guerra, $P h D^{6}$, \\ Francisco Pérez-Vázquez, PhD ${ }^{5}$, and Fidel Martinez-Gutierrez, PhD ${ }^{2,5}$
}

\begin{abstract}
${ }^{1}$ Hospital General de Subzona No.9, Instituto Mexicano del Seguro Social,Fray Juan Bautista de Mollinedo No 26, Rioverde, San Luis Potosí,Mexico; ${ }^{2}$ Posgrado en Ciencias Farmacobiológicas,Facultad deCiencias Químicas, Universidad Autónoma de San Luis Potosí,San Luis Potosí,Mexico; ${ }^{3}$ Research Unit,Colegio Mexicano deReumatología,MexicoCity,Mexico; ${ }^{4}$ CONACYTResearch Fellow,Coordinación parala Innovacióny Aplicación delaCienciaylaTecnología(CIACYT),Universidad Autónoma de San Luis Potosí,San Luis Potosí,Mexico; ${ }^{5}$ Centro de Investigación en Ciencias de la Salud y Biomedicina, Universidad Autónoma de San Luis Potosí, San Luis Potosí,Mexico; ${ }^{6}$ Laboratorio de BiotecnologíaMolecular,Facultad deCiencias Químicas, UniversidadAutónoma deSan LuisPotosí,San Luis Potosí,Mexico
\end{abstract}

BACKGROUND: Coronavirus disease 2019 (COVID-19) causes a mild illness in most cases; forecasting COVID19-associated mortality and the demand for hospital beds and ventilators are crucial for rationing countries' resources.

OBJECTIVE: To evaluate factors associated with the severity of COVID-19 in Mexico and to develop and validate a score to predict severity in patients with COVID19 infection in Mexico.

DESIGN: Retrospective cohort.

PARTICIPANTS: We included 1,435,316 patients with COVID-19 included before the first vaccine application in Mexico; 725,289 (50.5\%) were men; patient's mean age (standard deviation (SD)) was 43.9 (16.9) years; $21.7 \%$ of patients were considered severe COVID-19 because they were hospitalized, died or both.

MAIN MEASURES: We assessed demographic variables, smoking status, pregnancy, and comorbidities. Backward selection of variables was used to derive and validate a model to predict the severity of COVID-19.

KEY RESULTS: We developed a logistic regression model with 14 main variables, splines, and interactions that may predict the probability of COVID-19 severity (area under the curve for the validation cohort $=82.4 \%$ ).

CONCLUSIONS: We developed a new model able to predict the severity of COVID-19 in Mexican patients. This model could be helpful in epidemiology and medical decisions.

Keywords COVID-19 $\cdot$ Mortality $\cdot$ Hospitalization $\cdot$ Severity

$\mathrm{J}$ Gen Intern Med 37(3):624-31

DOI: $10.1007 / \mathrm{s} 11606-021-07235-0$

(c) Society of General Internal Medicine 2021

Received July 11, 2021

Published online January 7, 2022

\section{INTRODUCTION}

In December 2019, in a Wuhan animal market, clusters of patients with pneumonia were identified. The etiology of this pneumonia was a novel coronavirus: the severe acute respiratory syndrome coronavirus 2 (SARS-CoV-2) ${ }^{1-3}$. The World Health Organization announced the name of the illness as the coronavirus disease 19 (COVID-19). Up to June 7th, 2021, more than 173 million cases of COVID-19 have been confirmed worldwide, with over 3.5 million deaths recorded across the countries (Johns Hopkins University Web page) ${ }^{4}$. Latin-American countries, including Mexico, are among the highest number of deaths.

Forecasting COVID-19-associated mortality and the demand for hospital beds and ventilators are crucial for rationing countries' resources ${ }^{5}$. Number of people in critical conditions has compromised the healthcare capacity in some countries during the outbreak ${ }^{6}$.

Even though COVID-19 causes a mild illness in most cases (nearly $50-75 \%$ of positive to SARS-CoV-2 remain without symptoms) ${ }^{7,8}$, mortality and hospital admissions for COVID-19 are a burden in Mexico; moreover, approximately $10 \%$ of symptomatic patients will develop any of dyspnea, interstitial pneumonia, acute respiratory distress syndrome, or multiorgan dysfunction ${ }^{7}$. Advanced age, male gender, obesity, and the presence of non-communicable diseases, such as type 2 diabetes, hypertension, cardiovascular disease (CVD), chronic obstructive pulmonary disease (COPD), and cancer are factors associated with severe COVID-19, higher mortality, and, consequently, hospitalization ${ }^{2,9-13}$. In Mexico, almost 50\% of patients with COVID-19 reported at least one comorbidity ${ }^{14}$. Remarkably, $38.8 \%$ of patients with COVID-19 in Mexico were hospitalized, and the risk of hospitalization increases with the number of comorbidities ${ }^{15}$.

This study aimed to identify factors associated with severe COVID-19 (death or hospitalization) in Mexico and develop a model using readily available clinical variables to predict severe COVID-19. 


\section{MATERIALS AND METHODS}

\section{Study Design}

This retrospective cohort analysis uses the Mexican Epidemiological Surveillance System data for Viral Respiratory Diseases. We obtained all data from publicly available sources. Informed consent was not required as the information was de-identified.

\section{Data Collection}

We used free license data from the Mexican Epidemiological Surveillance System for Viral Respiratory Disease. In brief, all patients with COVID-19 symptoms who were tested for SARS-CoV-2 infection receive a medical questionary at the test time. This questionnaire includes demographic variables and comorbidities; moreover, all data for positive patients were updated daily if the patient was hospitalized or died (the patient was followed by the unit that made the test).

From the registered variables in the questionnaire, we selected the following for this study: demographic variables, smoking status, pregnancy, and comorbidities, including hypertension, type 2 diabetes, COPD, asthma, and others. In addition, we excluded variables reported after the SARSCoV-2 confirmation or after hospitalization by COVID-19 (intubation, pneumonia, admission to the intensive care unit). Severe COVID-19 was defined as death or requirement of hospitalization. Hospitalization, death, and recovery status are updated daily in the database.

We performed a summary analysis of data included up to June 7th, 2021. However, we selected patients included in the database for the model development before applying the first dose of the vaccine in Mexico (December 23rd, 2020) because of a potential modification of the vaccine in the severity of COVID-19 (Fig. 1, Supplementary material).

\section{Statistical Analysis}

Descriptive statistics were used to summarize the characteristics of all patients from derivation and validation cohorts. We expressed categorical variables as frequency and percentages; univariate comparison for categorical variables was performed with $X^{2}$ or Fisher's test.

We randomly selected $70 \%$ of observations to derive the logistic regression model (derivation cohort); the other 30\% of the patients were assigned to validate the model (validation cohort).

For this study, we considered severe COVID-19 if the patients were hospitalized or died. We developed a logistic regression model to estimate the probability of severe COVID-19.

All variables had missingness less than 5\% (supplementary material Table 1); for the model development and validation, we eliminated the patients with missing data for the variables included in the final model (supplementary material Table S1).

After confirming the absence of collinearity, we included all possible covariates to develop the model. We explored trends of variables with non-parametric regression models that describe the relationship between a response variable and the predictors without assuming a linear relationship among the variables. We began with an initial saturated logistic model including a flexible non-linear age effect with two-way interactions. Interactions were removed from the model (one by one) when the $p$-value was $>0.05$ (backward selection) up to maintain only the significant interactions variables; the main variables and splines were held in the model if there were significant interactions (including the non-significant primary variable). Finally, we estimated the probability of being hospitalized using the model. We conducted all statistical analysis with $\mathrm{R}$ version 4.0.4 (The $\mathrm{R}$ Foundation for Statistical Computing) and RStudio Version 1.3.1093 @ 2009-2020 RStudio, Inc.

\section{RESULTS}

The initial database included 7,134,254 patients with suspected COVID-19; 4,699,692 were negative to SARS-CoV-2 or were considered without data of COVID-19 (Fig. 1 in supplementary appendix). In R, we performed mining and cleaning data; for example, the database included pregnant patients less than 5 years or older than 50 (we corrected these values as non-pregnant). Moreover, male patients had missing data in the variable of pregnancy when the real value was non-pregnant.

A total of 2,434,562 patients were considered with the diagnosis of COVID-19 up to June 7th, 2021; mean age (SD) was 43.6 (17.1) years, 1,218,425 (50\%) were men, 456,909 (18.8\%) required hospitalization, and 228,828 patients (9.4\%) died; severe COVID-19, including hospitalized or death patients, occurred in 476,512 patients (19.6\%). Table 1 shows the frequency of smokers and comorbidities registered in the database according to the severity (all patients included up to June 7th, 2021).

We excluded 1,216,137 patients admitted in the database after December 23rd, 2020; therefore, the model development and validation database included 1,435,316 patients (Fig. 1 supplementary appendix). Table 2 shows the analyzed characteristics and the comparison between severe and non-severe patients with COVID-19 and the total cohort of patients before the vaccine in the univariate analysis. The proportion of indigenous patients, smokers, and with chronic conditions was significantly higher in severe than in nonsevere COVID-19 (Table 2); in contrast, pregnancy and asthma were more frequent in non-severe COVID-19 ("protective factors"). Figure 1 shows a univariable summary that describes the most substantial effect of chronic renal failure 
Table 1 Comparison of Severe and Non-severe Patients, Including All Patients up to June 7th, 2021
Table 2 Comparison of Patients with Severe and Nonsevere COVID-19 (Patients Admitted in the Database Before December 23rd, 2020)

\begin{tabular}{lllll}
\hline & Overall & Non-severe & Severe & $p$-value \\
\hline & $n=2,434,562$ & $1,958,050$ & 476,512 & \\
Sex = male (\%) & $1,218,425(50.0)$ & $934,326(47.7)$ & $284,099(59.6)$ & $<0.001$ \\
Age (mean (SD)) & $43.61(17.07)$ & $40.1(15.3)$ & $58.2(16.3)$ & $<0.001$ \\
Hospitalization (\%) & $456,909(18.8)$ & $0(0.0)$ & $456,909(95.9)$ & $<0.001$ \\
Indigenous (\%) & $20,052(0.9)$ & $13,661(0.7)$ & $6391(1.4)$ & $<0.001$ \\
Pregnancy (\%) & $15,650(0.6)$ & $12,824(0.7)$ & $2826(0.6)$ & $<0.001$ \\
Diabetes (\%) & $319,597(13.2)$ & $166,102(8.5)$ & $153,495(32.4)$ & $<0.001$ \\
COPD (\%) & $26,565(1.1)$ & $10,057(0.5)$ & $16,508(3.5)$ & $<0.001$ \\
Asthma (\%) & $52,784(2.2)$ & $43,375(2.2)$ & $9409(2.0)$ & $<0.001$ \\
Immunosuppression (\%) & $19,857(0.8)$ & $10,176(0.5)$ & $9681(2.0)$ & $<0.001$ \\
Hypertension (\%) & $415,287(17.1)$ & $233,790(12.0)$ & $181,497(38.3)$ & $<0.001$ \\
Other comorbidities (\%) & $46,640(1.9)$ & $24,844(1.3)$ & $21,796(4.6)$ & $<0.001$ \\
CVD (\%) & $37,237(1.5)$ & $17,470(0.9)$ & $19,767(4.2)$ & $<0.001$ \\
Obesity (\%) & $344,173(14.2)$ & $243,962(12.5)$ & $100,211(21.1)$ & $<0.001$ \\
CRF (\%) & $35,898(1.5)$ & $11,206(0.6)$ & $24,692(5.2)$ & $<0.001$ \\
Smoker (\%) & $177,712(7.3)$ & $142,361(7.3)$ & $35,351(7.5)$ & $<0.001$ \\
Deceased (\%) & $228,838(9.4)$ & $0(0.0)$ & $228,838(48.0)$ & $<0.001$ \\
\hline
\end{tabular}

Abbreviations: COPD chronic obstructive pulmonary disease, $C V D$ cardiovascular disease, $C R F$ chronic renal failure

\begin{tabular}{lllll}
\hline & Overall & Non-severe & Severe & $p$-value \\
\hline$n$ & $1,435,316$ & $1,124,126$ & 311,190 & \\
Sex = male (\%) & $725,289(50.5)$ & $537,214(47.8)$ & $188,075(60.4)$ & $<0.001$ \\
Age (mean (SD)) & $43.94(16.88)$ & $40.2(15.0)$ & $57.6(16.3)$ & $<0.001$ \\
Hospitalization (\%) & $297,964(20.8)$ & $0(0.0)$ & $297,964(95.7)$ & $<0.001$ \\
Indigenous (\%) & $13,181(1.0)$ & $8668(0.8)$ & $4513(1.5)$ & $<0.001$ \\
Pregnancy (\%) & $9304(0.6)$ & $7484(0.7)$ & $1820(0.6)$ & $<0.001$ \\
Diabetes (\%) & $201,755(14.1)$ & $100,053(8.9)$ & $101,702(32.9)$ & $<0.001$ \\
COPD (\%) & $17,820(1.2)$ & $6506(0.6)$ & $11,314(3.7)$ & $<0.001$ \\
Asthma (\%) & $34,323(2.4)$ & $27,732(2.5)$ & $6591(2.1)$ & $<0.001$ \\
Immunosuppression (\%) & $13,541(0.9)$ & $6676(0.6)$ & $6865(2.2)$ & $<0.001$ \\
Hypertension (\%) & $259,529(18.1)$ & $141,363(12.6)$ & $118,166(38.2)$ & $<0.001$ \\
Other comorbidities (\%) & $30,130(2.1)$ & $15,507(1.4)$ & $14,623(4.7)$ & $<0.001$ \\
CVD (\%) & $24,870(1.7)$ & $11,529(1.0)$ & $13,341(4.3)$ & $<0.001$ \\
Obesity (\%) & $226,154(15.8)$ & $157,725(14.1)$ & $68,429(22.1)$ & $<0.001$ \\
CRF (\%) & $23,831(1.7)$ & $7165(0.6)$ & $16,666(5.4)$ & $<0.001$ \\
Smoker (\%) & $107,398(7.5)$ & $83,621(7.5)$ & $23,777(7.7)$ & $<0.001$ \\
Deceased (\%) & $147,180(10.3)$ & $0(0.0)$ & $147,180(47.3)$ & $<0.001$ \\
\hline
\end{tabular}

Abbreviations: $C O P D$ chronic obstructive pulmonary disease, $C V D$ cardiovascular disease, $C R F$ chronic renal failure
(CRF), chronic obstructive pulmonary disease (COPD), and cardiovascular disease (CVD) on the severity of COVID-19.

In the model development (derivation cohort-960,201 patients), we explored trends with non-parametric regression (with loess fit). As a result, we obtained the age distribution: Fig. 2 in the supplementary appendix shows the association between age and the probability of severe COVID-19 in different statuses of the categorical variables; these figures showed non-linearity of age and interactions between age and categorical variables. We modeled age as a restricted cubic spline (or natural splines) with 5 degrees of freedom.

In the initial analysis, we eliminated the observations with missing data (Table 1 in supplementary material); the initial saturated logistic regression model allows for a flexible nonlinear effect of age and two-way interactions. We reduced the model by removing the interactions when the $p$-value 
Fig. 1 Univariable summaries of data used for development and validation. The figure shows missing data by variables. Abbreviations: COPD, chronic obstructive pulmonary disease; CVD, cardiovascular disease; $\mathrm{CRF}$, chronic renal failure; Dead_Hosp, dead or hospitalization or both

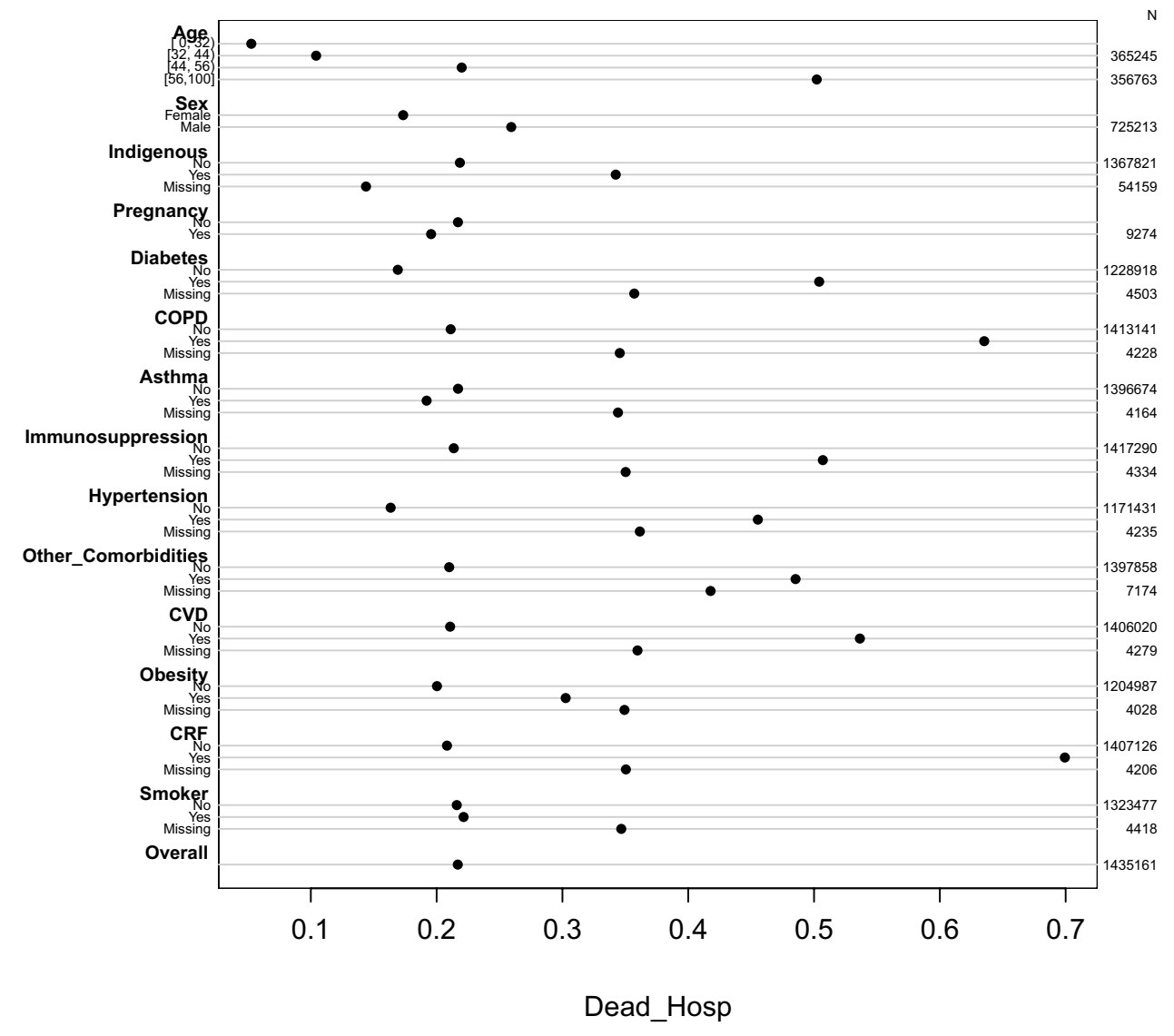

was $>0.05$ (backward selection). Because of the non-linearity, the log-odds is the best form to express the results shown in Fig. 2; Fig. 2 shows the effect on log odds of smoker men and women is higher in older people. Figure 2 also shows the impact of asthma according to age in men and women. These figures show the only reduction in log odds of severity for younger people. Still, there is a notorious increase in the odds of severity in people older than 25 years for smokers.

Model discrimination was good with an area under the curve of $82.5 \%$ in the derivation, and $82.4 \%$ in the validation cohort (Supplementary material 1, Fig. 3); the calibration plot showed a good agreement between predicted and observed risks (Fig. 4).

The final model-with 14 main variables, splines, and interactions - is shown in Fig. 3 supplementary material. In addition, this supplemental material shows the beta coefficients, standard errors, and $p$-values; with these values, we developed an application in shiny for R and RStudio to calculate the probability of COVID-19 severity at: https:// marcomtzmtz.shinyapps.io/HospitalizationCOVID/.

\section{DISCUSSION}

This study focused on creating a score that helps distinguish patients with a severe COVID-19 in México using accessible clinical variables. We report developing a model that predicts severe-COVID-19 based on readily available clinical variables asked by the Mexican Epidemiological Surveillance System for Viral Respiratory Diseases. In Mexican patients with COVID-19, 14 variables can predict severe COVID-19 with a well-calibrated model with proper discrimination.

Definition of severe COVID-19 may be different across authors: severe illness usually begins with dyspnea and hypoxemia, and these patients commonly meet criteria for ARDS requiring hospitalization or concluding in death ${ }^{16}$. We considered severe COVID-19 when patients died or were hospitalized because of the characteristics of our database (these data were daily updated for all patients).

Since the pandemic began, all countries have made substantial efforts to ascertain optimal strategies for the medical attention of the outbreak. COVID-19 pandemic represents a significant public health challenge for the world ${ }^{17,18}$. Even though $80 \%$ of patients with COVID-19 have a mild illness ${ }^{2}$, estimating required hospital beds is critical to scaling the health services capacity ${ }^{5}$. In the Latin American region, Mexico is one of the countries with the highest number of deaths cases of COVID-19 ${ }^{4}$. The Mexican health sector has 49,083 hospital beds, 2,446 intensive care unit beds, and 5,523 mechanical ventilators ${ }^{19}$.

The probability of COVID-19 severity increases in known factors: obesity ${ }^{20}$, type- 2 diabetes ${ }^{21}$, coronary heart disease ${ }^{21}$, systemic hypertension ${ }^{22}$, and chronic renal failure ${ }^{23}$. Mexico is facing the double burden of malnutrition and the prevalence of chronic diseases, including type 2 diabetes, obesity, and hypertension; these diseases caused almost half of all deaths in 
A
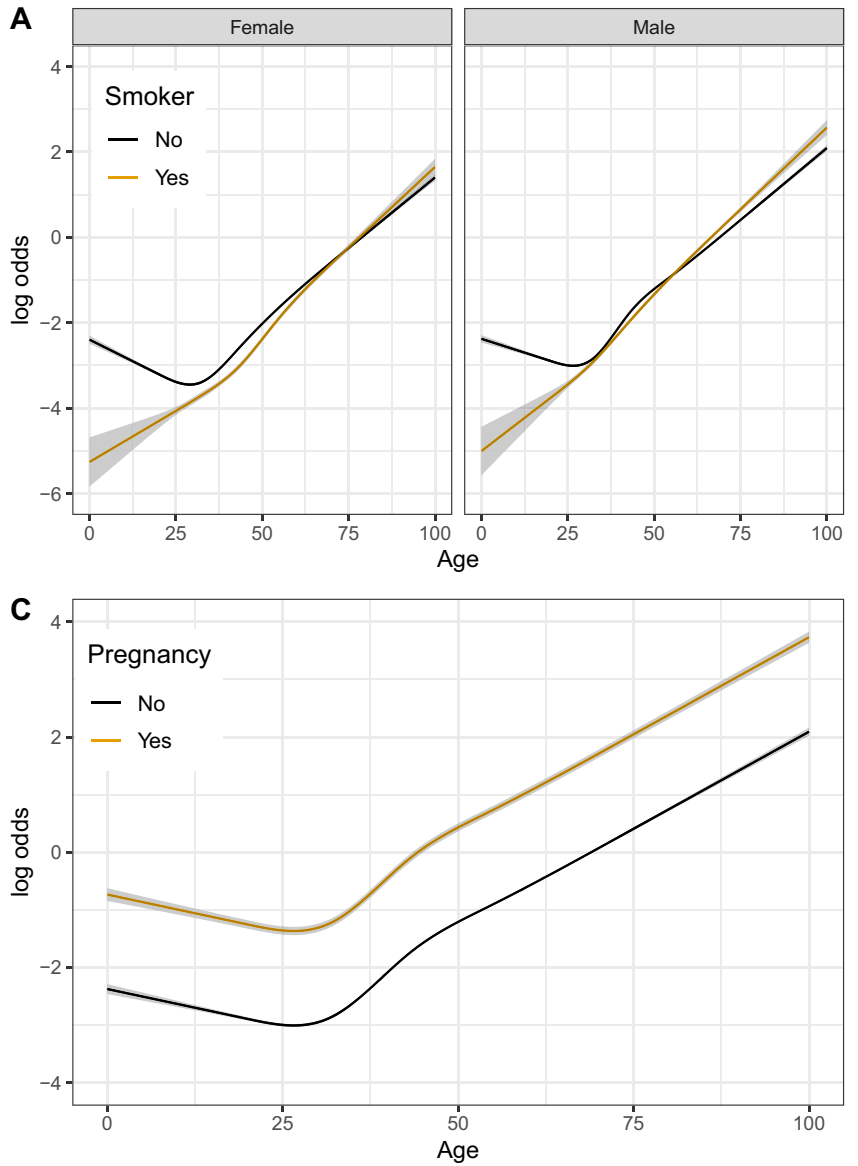

B
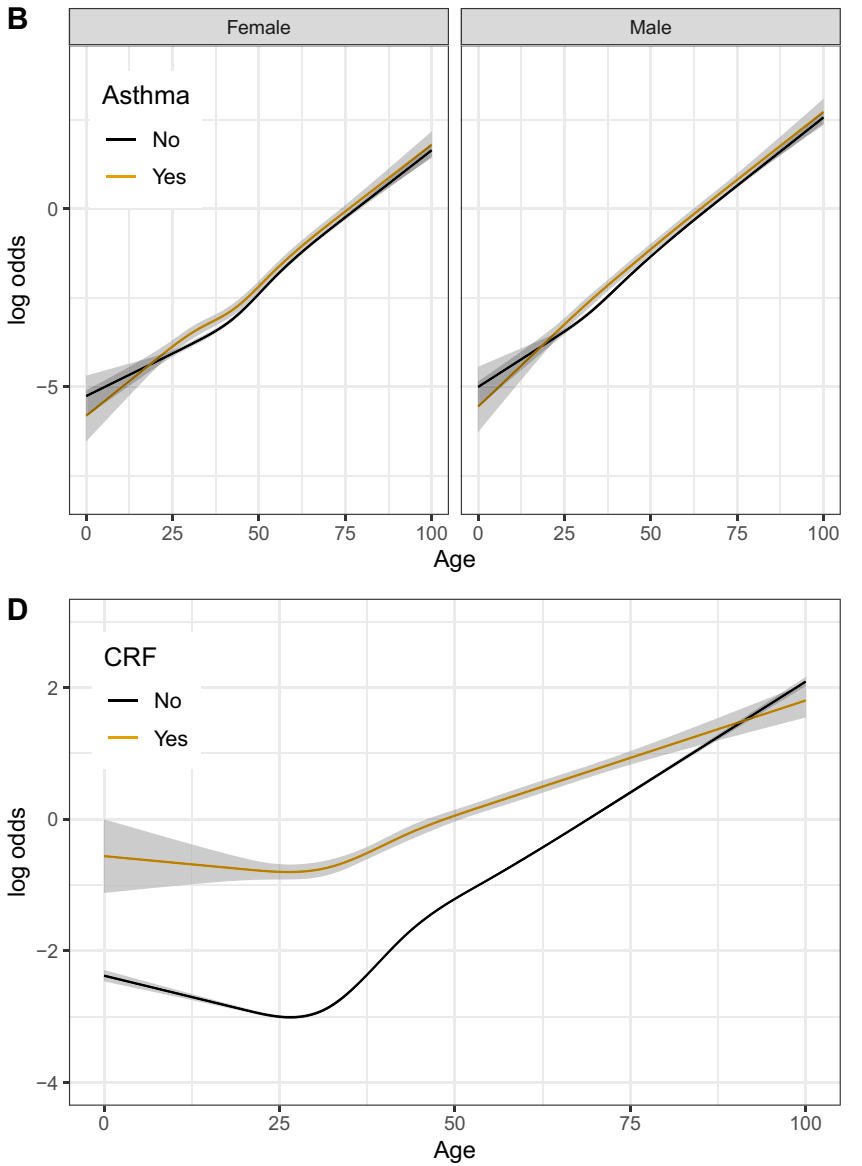

Fig. 2 Predictions based on the model for smoking, asthma, pregnancy, and chronic renal failure (CRF)

Mexico before COVID-19 and are related to COVID-19 severe upon admission in Mexican patients ${ }^{24}$.

In our study, men have a higher risk of severe COVID19 than women; this finding is consistent with the study reported by Jin et al., who describe worse outcomes and death for men ${ }^{25}$.

Moreover, sex and age play a central role in the expression of membrane-bound angiotensin-converting enzyme 2 , also associated with a severe COVID-19 ${ }^{26}$.

Our study shows an increased probability of severity for pregnant patients. The hospitalization odds of pregnancy increased in our study, probably related more to the monitoring of the pregnant patient than to the severity of the disease. Case series inform lower or no mortality of pregnant patients with COVID-19 compared to other coronavirus infections (severe acute respiratory syndrome (SARS) and the Middle East respiratory syndrome (MERS) ${ }^{27,28}$. A systematic review of 77 studies showed that pregnant women with COVID-19 have an increased risk of admission to an intensive care unit, preterm birth, and reported risk factors for severe COVID-19, including advanced maternal age, high body mass index, and pre-existing comorbidities ${ }^{29}$.

In our study, in the univariate analysis, asthma reduces the odds of severity. Multivariate analysis, including interactions and non-linear relationships of age, shows that asthma increases the odds of severity, particularly in older men. Mahdavinia et al. found prolonged intubation in patients with asthma and COVID-19, but asthma was not associated with a higher rate of acute respiratory distress syndrome nor hospitalization ${ }^{30}$.

Indigenous people had a higher odd for hospitalization in our study; we consider this fact an indirect marker of social factors of the Mexican indigenous population with potential poor access to health services ${ }^{31}$. Moreover, other authors have reported a correlation between mortality and healthcare availability ${ }^{32,33}$. Mortality for COVID-19 has been reported as higher in low-income neighborhoods and rural communities in Ecuador and Mexico ${ }^{34,35}$.

There are prediction models for the diagnosis and prognosis of COVID-19; some of them require studies like chest tomography, or their prediction could be unreliable when applied in daily practice ${ }^{36}$. DeCaprio et al. developed a vulnerability index but with other conditions different to COVID-19 ${ }^{37}$; Gong et al. developed a tool to predict severe COVID-19, but the weaknesses of this study were the small sample size and requirement of laboratory tests to predict the severity ${ }^{38}$. In Spain, MartínRodriguez et al. showed that prehospital lactate improved the National Early Warning Score 2 (NEWS2) capacity to detect the mortality risk ${ }^{39}$. The Epic Deterioration Index in the United 

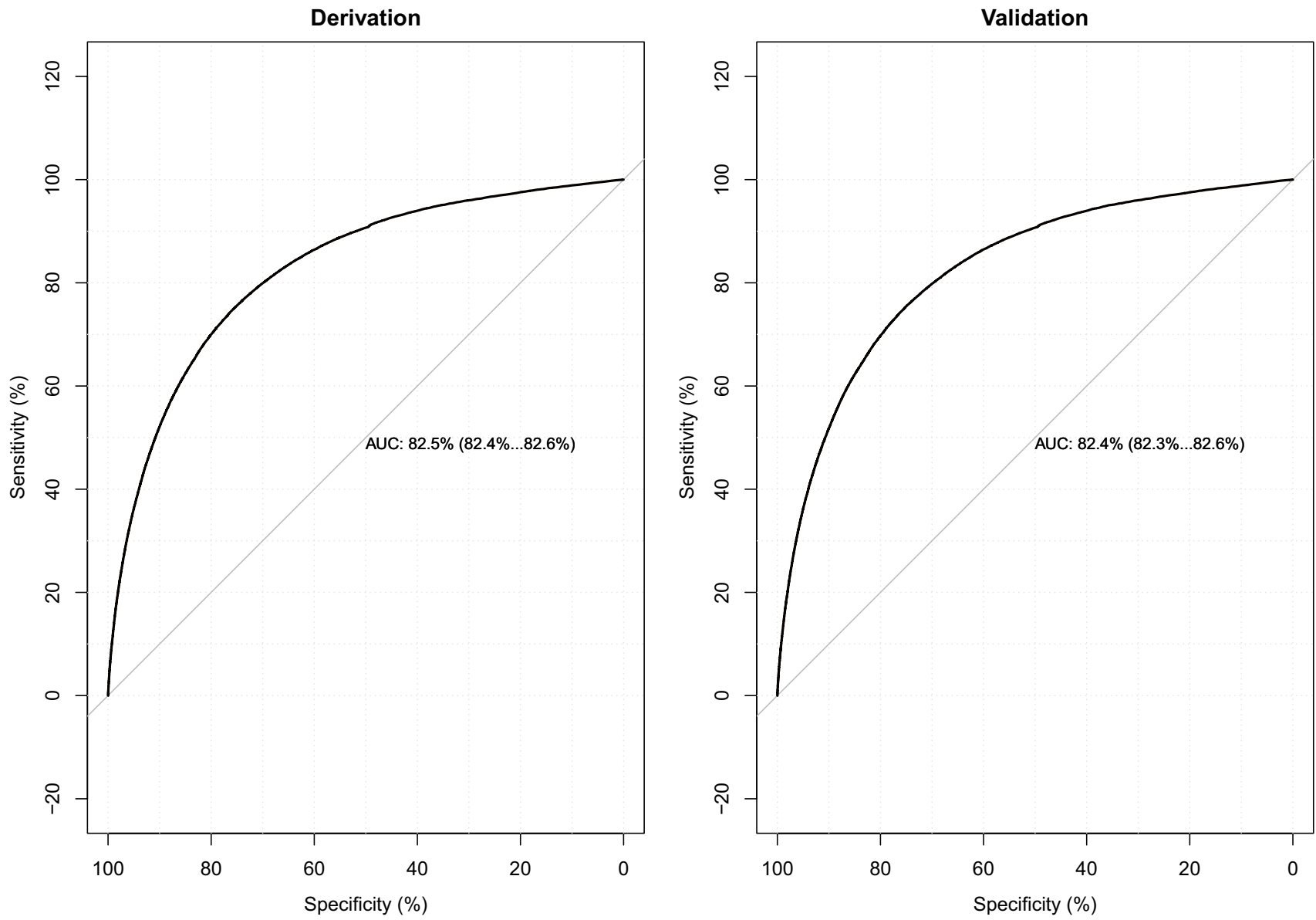

Fig. 3 Receiver operating characteristic (ROC) curves for derivation and validation cohorts

States identifies high and low risks patients; but, this index was developed in 392 hospitalized patients with low sensitivity in contrast to our study that include hospitalized and non-hospitalized large cohort ${ }^{40}$.
We generated a reliable index that can be applied easily in clinical practice and predict the severity of COVID19 patients. We suggest our score for a strict follow-up of patients who do not require hospitalization at the moment
Fig. 4 Calibration plot. Agreement between predicted and observed hospitalization risks. (Derivation cohort)
Calibration plot

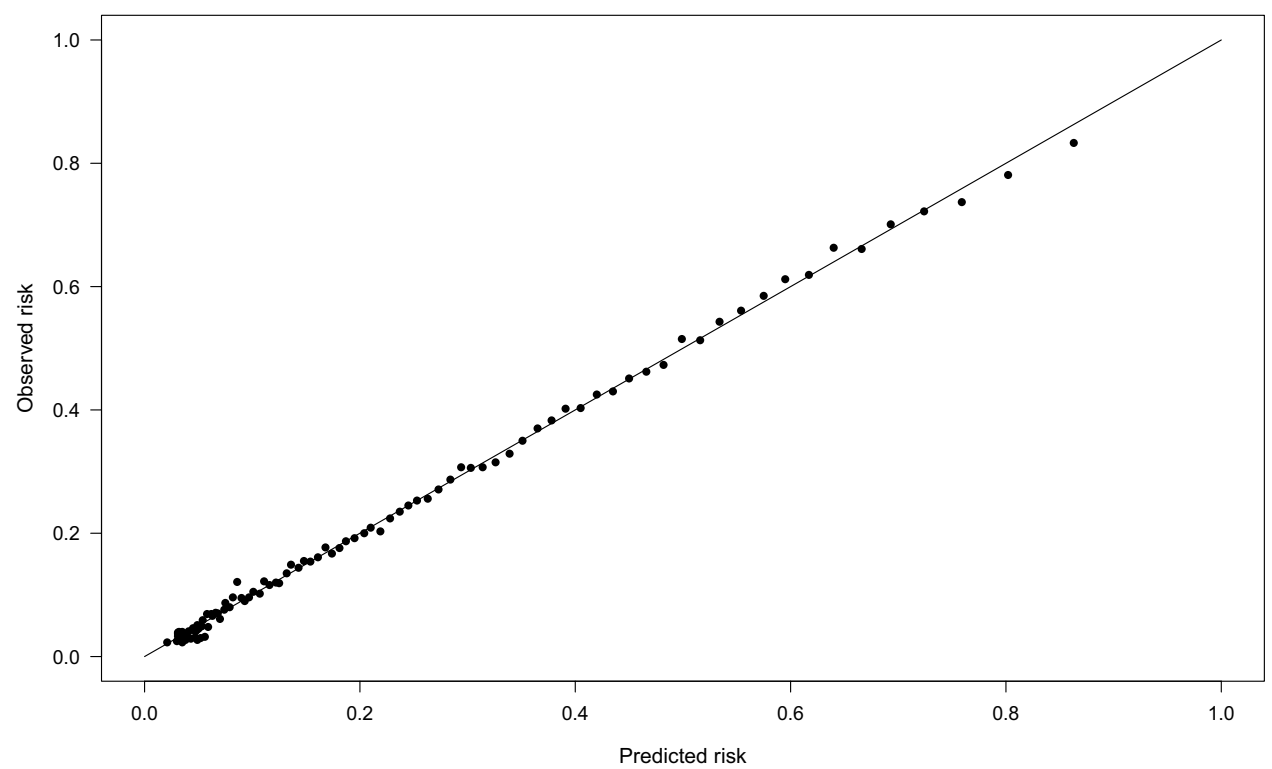


of diagnosis but have a high risk of severity. For example, a 51-year-old man with type 2 diabetes, systemic hypertension, chronic renal failure, and obesity scored $79.4 \%$ of the probability of being a severe COVID-19. Maybe this patient does not require hospital admission at the time of diagnosis, but, according to our score, more than $70 \%$ of patients in this group will require hospitalization or die. We suggest a strict follow-up of his symptoms and tight laboratory work. Another utility of our study is to prioritize the uses of vaccines or specific medications for COVID-19. For example, the group of 35-year-old diabetic patients and chronic renal failure have a $65.8 \%$ probability of being a severe COVID19 , compared with patients of 60 years without comorbidities having a $35.7 \%$ of being a severe COVID- 19 .

The strengths of our study include the number of patients and the information contained in the Mexican Epidemiological Surveillance System for Viral Respiratory Diseases. It helped us to develop a model that concluded in a helpful score to predict hospitalization. We provide a practical and inexpensive tool; our conclusions are robust because of the large sample size and validation. In Mexico, general doctors affront decisions about the care of patients with suspected COVID-19 in the community. Still, without access to a laboratory or radiological tests, this fact is typical for developing countries which our model could be helpful after validation for each country.

We consider that our score improves the prediction of Bello-Chavolla et al. ${ }^{41}$ : we included all possible interactions and the non-linearity of age that must be included in every model. Furthermore, because of the robust statistics used in our model, we demonstrated the spurious "protective effect of asthma" in the severity of COVID-19 ${ }^{41,42}$.

A limitation of our study is the retrospective design. We did not have the information about the previous treatment and the activity of comorbidities, such as asthma or hypertension. Another limitation is that some clinical data were selfreported or reported by relatives but not confirmed as medical records were unavailable to corroborate the information.

Another limitation of our study is the no inclusion of laboratory parameters: there are some like hemogram indexes ${ }^{43,44}$, hepcidin, and ferritin ${ }^{45}$ that may have a role as valuable tools in the prediction of COVID-19 severity; however, we considered our score with a higher utility in developing countries and epidemiology because it does not require to have laboratory tests.

We have no information on which variants affect our patients; according to the date of inclusion, low or no patients with delta variant were not included, which is another limitation. Therefore, our score must be validated with the newer variants of SARS-CoV-2.

In summary, we developed and validated an inexpensive, readily available score to predict severe COVID-19 in Mexican patients with the SARS-CoV-2 infection. With this score, the clinician may suggest a stricter follow-up or implement re-evaluation for patients who were not admitted at the first examination but have a high risk of severe COVID-19. Thus, this model could have a crucial role in epidemiology and clinician's decision-making.

Corresponding Author: Marco Ulises Martinez-Martínez, MD, MSc; Hospital General de Subzona No. 9, Instituto Mexicano del Seguro Social, Fray Juan Bautista de Mollinedo No 26, Rioverde, San Luis Potosí, Mexico (e-mail: marcomtzmtz@hotmail.com).

Supplementary Information The online version contains supplementary material available at https://doi.org/10.1007/ s11606-021-07235-0.

\section{Declarations}

Conflict of Interest All authors declare that they have no any conflict of interest.

\section{References}

1. Zhang N, Li C, Hu Y, Li K, Liang J, Wang L, et al. Current development of COVID-19 diagnostics, vaccines and therapeutics. Microbes Infect. 2020;22(6-7):231-5. doi:https://doi.org/10.1016/j.micinf. 2020.05.001

2. Epidemiology Working Group for Ncip Epidemic Response CCfDC, Prevention. [The epidemiological characteristics of an outbreak of 2019 novel coronavirus diseases (COVID-19) in Chinal. Zhonghua Liu Xing Bing Xue Za Zhi. 2020;41(2):145-51. doi:https://doi.org/10.3760/ cma.j.issn.0254-6450.2020.02.003

3. Zhu N, Zhang D, Wang W, Li X, Yang B, Song J, et al. A novel coronavirus from patients with pneumonia in China, 2019. N Engl J Med. 2020;382(8):727-33. doi:https://doi.org/10.1056/NEJMoa2001017

4. Johns Hopkins University of M. COVID-19 Map - Johns Hopkins Coronavirus Resource Center. 2021.

5. Moghadas SM, Shoukat A, Fitzpatrick MC, Wells CR, Sah P, Pandey A, et al. Projecting hospital utilization during the COVID19 outbreaks in the United States. Proc Natl Acad Sci U S A. 2020;117(16):9122-6. doi:https://doi.org/10.1073/pnas.2004064117

6. Requia WJ, Kondo EK, Adams MD, Gold DR, Struchiner CJ. Risk of the Brazilian health care system over 5572 municipalities to exceed health care capacity due to the 2019 novel coronavirus (COVID-19). Sci Total Environ. 2020;730:139144. doi:https://doi.org/10.1016/j. scitotenv.2020.139144

7. Aktas G. A comprehensive review on rational and effective treatment strategies against an invisible enemy; SARS Cov-2 infection. Experimental Biomedical Research. 2020;3(4):293-311. doi:https://doi.org/ $10.30714 /$ j-ebr.2020463629

8. Lavezzo E, Franchin E, Ciavarella C, Cuomo-Dannenburg G, Barzon L, Del Vecchio C, et al. Suppression of a SARS-CoV-2 outbreak in the Italian municipality of Vo'. Nature. 2020;584(7821):425-9. doi:https://doi.org/10.1038/s41586-020-2488-1

9. Huang $\mathbf{R}$, Zhu L, Xue L, Liu L, Yan X, Wang J, et al. Clinical findings of patients with coronavirus disease 2019 in Jiangsu province, China: a retrospective, multi-center study. PLoS Negl Trop Dis. 2020; 14(5):e0008280. doi:https://doi.org/10.1371/journal.pntd. 0008280

10. Sun H, Ning R, Tao Y, Yu C, Deng X, Zhao C, et al. Risk factors for mortality in 244 older adults with COVID-19 in Wuhan, China: a retrospective study. J Am Geriatr Soc. 2020;68(6):E19-E23. doi:https: // doi.org/10.1111/jgs. 16533

11. Zhou F, Yu T, Du R, Fan G, Liu Y, Liu Z, et al. Clinical course and risk factors for mortality of adult inpatients with COVID-19 in Wuhan, China: a retrospective cohort study. Lancet. 2020;395(10229): 105462. doi:https://doi.org/10.1016/S0140-6736(20)30566-3

12. Fang L, Karakiulakis G, Roth M. Are patients with hypertension and diabetes mellitus at increased risk for COVID-19 infection? The Lancet Respiratory Medicine. 2020;8(4):e21-e. doi:https://doi.org/ $10.1016 / \mathrm{s} 2213-2600(20) 30116-8$ 
13. Clark A, Jit M, Warren-Gash C, Guthrie B, Wang HHX, Mercer SW, et al. Global, regional, and national estimates of the population at increased risk of severe COVID-19 due to underlying health conditions in 2020: a modelling study. Lancet Glob Health. 2020;8(8):e1003-e17. doi:https://doi.org/10.1016/S2214-109X(20)30264-3

14. Hernandez-Galdamez DR, Gonzalez-Block MA, Romo-Duenas DK, Lima-Morales R, Hernandez-Vicente IA, Lumbreras-Guzman M, et al. Increased risk of hospitalization and death in patients with COVID-19 and pre-existing noncommunicable diseases and modifiable risk factors in Mexico. Archives of medical research. 2020. doi:https://doi.org/10.1016/j.arcmed.2020.07.003

15. Kammar-Garcia A, Vidal-Mayo JJ, Vera-Zertuche JM, LazcanoHernandez M, Vera-Lopez 0 , Segura-Badilla 0 , et al. Impact of comorbidities in Mexican Sars-Cov-2-positive patients: a retrospective analysis in a national cohort. Revista de investigacion clinica; organo del Hospital de Enfermedades de la Nutricion. 2020;72(3):151-8. doi:https://doi.org/10.24875/RIC.20000207

16. Berlin DA, Gulick RM, Martinez FJ. Severe Covid-19. N Engl J Med. 2020;383(25):2451-60. doi:https://doi.org/10.1056/NEJMc p2009575

17. van Halem $\mathbf{K}$, Cox $\mathbf{J}$, Messiaen $\mathbf{P}$, Pat $\mathbf{K}$, Declercq $\mathbf{C}$, Meersman A, et al. Care for adult non-ICU Covid-19 patients: early experiences from a Belgian tertiary care centre. Neth J Med. 2020;78(3):111-5.

18. Medeiros EAS. Challenges in the fight against the Covid-19 pandemic in university hospitals. Rev Paul Pediatr. 2020;38:e2020086. doi:https://doi.org/10.1590/1984-0462/2020/38/2020086

19. Nelly T. Insuficiente la infraestructura hospitalaria en México para COVID-19.

20. Chiappetta S, Sharma AM, Bottino v, Stier C. COVID-19 and the role of chronic inflammation in patients with obesity. Int $\mathrm{J}$ Obes (Lond). 2020;44(8):1790-2. doi:https://doi.org/10.1038/ s41366-020-0597-4

21. Phua J, Weng L, Ling L, Egi M, Lim CM, Divatia JV, et al. Intensive care management of coronavirus disease 2019 (COVID19): challenges and recommendations. Lancet Respir Med. 2020;8(5):506-17. doi:https://doi.org/10.1016/S2213-2600(20) 30161-2

22. Chen T, Wu D, Chen H, Yan W, Yang D, Chen G, et al. Clinical characteristics of 113 deceased patients with coronavirus disease 2019: retrospective study. BMJ. 2020;368:m1091. doi:https://doi. org/10.1136/bmj.m1091

23. Ke C, Wang $\mathbf{Y}$, Zeng $\mathbf{X}$, Yang C, Hu Z. 2019 Novel coronavirus disease (COVID-19) in hemodialysis patients: a report of two cases. Clin Biochem. 2020;81:9-12. doi:https://doi.org/10.1016/j.clinb iochem.2020.04.008

24. Alcalde-Rabanal JE, Orozco-Nunez E, Espinosa-Henao OE, Arredondo-Lopez A, Alcayde-Barranco L. The complex scenario of obesity, diabetes and hypertension in the area of influence of primary healthcare facilities in Mexico. PLoS One. 2018;13(1):e0187028. doi:https://doi.org/10.1371/journal.pone.0187028

25. Jin JM, Bai P, He W, Wu F, Liu XF, Han DM, et al. Gender differences in patients with COVID-19: focus on severity and mortality. Front Public Health. 2020;8:152. doi:https://doi.org/10.3389/ fpubh.2020.00152

26. Sward P, Edsfeldt A, Reepalu A, Jehpsson L, Rosengren BE, Karlsson MK. Age and sex differences in soluble ACE2 may give insights for COVID-19. Crit Care. 2020;24(1):221. doi:https://doi. org/10.1186/s13054-020-02942-2

27. Schwartz DA. An analysis of 38 pregnant women with COVID-19, their newborn infants, and maternal-fetal transmission of SARSCoV-2: maternal coronavirus infections and pregnancy outcomes. Arch Pathol Lab Med. 2020;144(7):799-805. doi:https://doi.org/10. 5858/arpa.2020-0901-SA

28. Chen S, Liao E, Cao D, Gao Y, Sun G, Shao Y. Clinical analysis of pregnant women with 2019 novel coronavirus pneumonia. J Med Virol. 2020;92(9):1556-61. doi:https://doi.org/10.1002/jmv.25789

29. Allotey J, Stallings E, Bonet M, Yap M, Chatterjee S, Kew T, et al. Clinical manifestations, risk factors, and maternal and perinatal outcomes of coronavirus disease 2019 in pregnancy: living systematic review and meta-analysis. Bmj. 2020;370:m3320. doi:https://doi. org/10.1136/bmj.m3320

30. Mahdavinia M, Foster KJ, Jauregui E, Moore D, Adnan D, AndyNweye AB, et al. Asthma prolongs intubation in COVID-19. J Allergy Clin Immunol Pract. 2020;8(7):2388-91. doi:https://doi.org/10. 1016/j.jaip.2020.05.006
31. Diaz de Leon-Martinez L, de la Sierra-de la Vega L, Palacios-Ramirez A, Rodriguez-Aguilar M, Flores-Ramirez R. Critical review of social, environmental and health risk factors in the Mexican indigenous population and their capacity to respond to the COVID-19. Sci Total Environ. 2020;733:139357. doi:https://doi.org/10.1016/j.scito tenv.2020.139357

32. Lai CC, Wang CY, Wang YH, Hsueh SC, Ko WC, Hsueh PR. Global epidemiology of coronavirus disease 2019 (COVID-19): disease incidence, daily cumulative index, mortality, and their association with country healthcare resources and economic status. Int J Antimicrob Agents. 2020;55(4):105946. doi:https://doi.org/10.1016/j.ijantimicag.2020.105946

33. Ji Y, Ma Z, Peppelenbosch MP, Pan Q. Potential association between COVID-19 mortality and health-care resource availability. The Lancet Global Health. 2020;8(4):e480-e. doi:https://doi.org/10.1016/s2214109x(20)30068-1

34. Del Brutto OH, Costa AF, Mera RM, Recalde BY, Bustos JA, Garcia HH. SARS-CoV-2 in rural Latin America. A population-based study in coastal Ecuador. Clin Infect Dis. 2020. doi:https://doi.org/10.1093/ cid/ciaa 1055

35. Friedman J, Calderon-Villarreal A, Bojorquez I, Vera Hernandez C, Schriger D, Tovar Hirashima E. Excess out-of-hospital mortality and declining oxygen saturation: the sentinel role of EMS data in the COVID-19 crisis in Tijuana, Mexico. medRxiv : the preprint server for health sciences. 2020. doi:https://doi.org/10.1101/2020.05.13. 20098186

36. Wynants L, Van Calster B, Collins GS, Riley RD, Heinze G, Schuit E, et al. Prediction models for diagnosis and prognosis of covid-19 infection: systematic review and critical appraisal. BMJ. 2020;369:m1328. doi:https://doi.org/10.1136/bmj.m1328

37. DeCapprio D, Gartner J, McCall CJ, Burgess T, Kothari S, Sayed S. Building a COVID-19 Vulnerability Index. medRxiv. 2020:2020.03.16.20036723-2020.03.16. doi:https://doi.org/10. $1101 / 2020.03 .16 .20036723$

38. Gong J, Ou J, Giu X, Jie Y, Chen Y, Yuan L, et al. A tool for early prediction of severe coronavirus disease 2019 (COVID-19): a multicenter study using the risk nomogram in Wuhan and Guangdong, China. Clin Infect Dis. 2020;71(15):833-40. doi:https://doi.org/10. 1093/cid/ciaa443

39. Martin-Rodriguez F, Lopez-Izquierdo R, Delgado Benito JF, Sanz-Garcia A, Del Pozo Vegas C, Castro Villamor MA, et al. Prehospital point-of-care lactate increases the prognostic accuracy of national early warning score 2 for early risk stratification of mortality: results of a multicenter, observational study. J Clin Med. 2020;9(4). doi:https://doi.org/10.3390/jcm9041156

40. Singh K, Valley TS, Tang S, Li BY, Kamran F, Sjoding MW, et al. Evaluating a widely implemented proprietary deterioration index model among hospitalized patients with COVID-19. Ann Am Thorac Soc. 2021;18(7): 1 129-37. doi:https://doi.org/10.1513/AnnalsATS. 202006-6980C

41. Bello-Chavolla OY, Bahena-Lopez JP, Antonio-Villa NE, VargasVazquez A, Gonzalez-Diaz A, Marquez-Salinas A, et al. Predicting mortality due to SARS-CoV-2: a mechanistic score relating obesity and diabetes to COVID-19 outcomes in Mexico. J Clin Endocrinol Metab. 2020;105(8). doi:https://doi.org/10.1210/clinem/dgaa346

42. Parra-Bracamonte GM, Lopez-Villalobos N, Parra-Bracamonte FE. Clinical characteristics and risk factors for mortality of patients with COVID-19 in a large data set from Mexico. Ann Epidemiol. 2020;52:93-8 e2. doi:https://doi.org/10.1016/j.annepidem.2020. 08.005

43. Aktas G. Hematological predictors of novel Coronavirus infection. Rev Assoc Med Bras (1992). 2021;67Suppl 1(Suppl 1):1-2. doi:https://doi.org/10.1590/1806-9282.67.Suppl1.20200678

44. Liu Y, Du X, Chen J, Jin Y, Peng L, Wang HHX, et al. Neutrophilto-lymphocyte ratio as an independent risk factor for mortality in hospitalized patients with COVID-19. J Infect. 2020;81(1):e6-e12. doi:https://doi.org/10.1016/j.jinf.2020.04.002

45. Zhou C, Chen Y, Ji Y, He X, Xue D. Increased serum levels of hepcidin and ferritin are associated with severity of COVID-19. Med Sci Monit. 2020;26:e926178. doi:https://doi.org/10.12659/MSM.926178

Publisher's Note Springer Nature remains neutral with regard to jurisdictional claims in published maps and institutional affiliations. 